\title{
Red enhances human performance in contests
}

\section{Signals biologically attributed to red coloration in males may operate in the arena of combat sports.}

Red coloration is a sexually selected, testosterone-dependent signal of male quality in a variety of animals $\mathrm{s}^{1-5}$, and in somenon-human species a male's dominance can be experimentally increased by attaching artificial red stimuli'. Here we show that a similar effect can influence the outcome of physical contests in humans - across a range of sports, we find that wearing red is consistently associated with a higher probability of winning. These results indicate not only that sexual selection may have influenced the evolution of human response to colours, but also that the colour of sportswear needs to be taken into account to ensure a level playing field in sport.

Although other colours are also present in animal displays, it is specifically the presence and intensity of red coloration that correlates with male dominance and testosterone levels $s^{3-5}$. In humans, anger is associated with a reddening of the skin due to increased blood flow $^{7,8}$, whereas fear is associated with increased pallor in similarly threatening situations'. Hence, increased redness during aggressive interactions may reflect relative dominance. Because artificial stimuli can exploit innate responses to natural stimuli $i^{6,10}$, we tested whether wearing red might influence the outcome of physical contests in humans.

In the 2004 Olympic Games, contestants in four combat sports (boxing, tae kwon do, Greco-Roman wrestling and freestyle wrestling) were randomly assigned red or blue outfits (or body protectors). If colour has no effect on the outcome of contests, the number of winners wearing red should be statistically indistinguishable from the number of winners wearing blue. However, we found that for all four competitions, there is a consistent and statistically significant pattern in which contestants wearing red win more fights $\left(\chi^{2}=4.19\right.$, d.f. $=1, P=0.041$; Fig. 1a). This result is remarkably consistent across rounds in each competition, with 16 of 21 rounds having more red than blue winners, and only four rounds having more blue winners (sign test, $P=0.012)$. The effect is the same across the weight classes in each sport: 19 of 29 classes had more red winners, with onlysix classes having more blue winners (sign test, $P=0.015$ ). (For methods, see supplementary information.)

Given the undoubted role of other factors, such as skill and strength, it is likely that the red advantage will determine the outcome
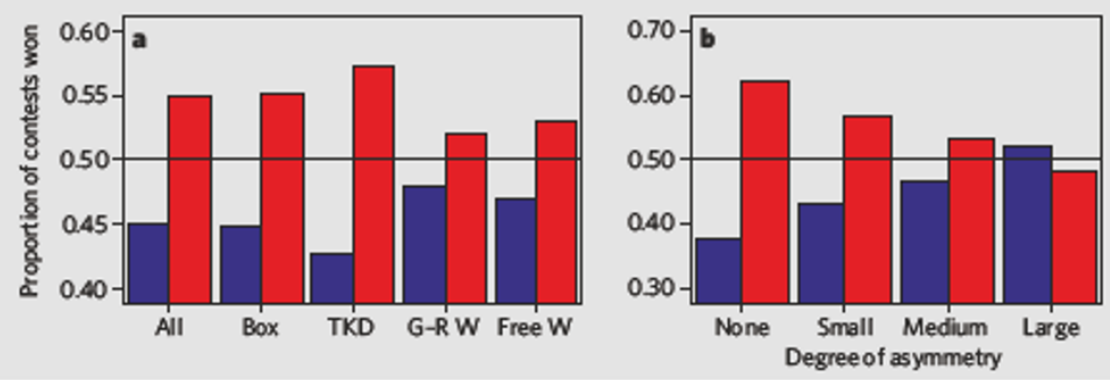

Figure 1 | Influence of colour of sporting attire on the outcome of competitive sports. a, Proportion of contests in Olympic combat sports won by competitors wearing red (right bars) or blue (left bars) outfits for all sports combined and for the individual sports of boxing (Box), tae kwon do (TKD), Greco-Roman wrestling (G-R W) and freestyle wrestling (Free W). b, Proportion of contests won by competitors wearing red or blue given different degrees of relative ability (asymmetry) in the two competitors in each bout. No significant differences exist between the number of red and blue wins for contests with small $\left(\chi^{2}=2.21\right.$, d.f. $\left.=1, P=0.14\right)$, medium $\left(\chi^{2}=0.47\right.$, d.f. $\left.=1, P=0.50\right)$ or large asymmetries $\left(\chi^{2}=0.21\right.$, d.f. $\left.=1, P=0.64\right)$ in competitive ability. Black lines at 0.5 indicate the expected proportion of wins by red or blue under the null hypothesis that colour has no effect on contest outcomes. For details of data collection and analyses, see supplementary information.

only in relatively symmetric contests. That is, wearing red presumably tips the balance between losing and winning only when other factors are fairly equal. We found that this is indeed the case: only in contests between individuals of similar ability were there significantly more red than blue winners $\left(\chi^{2}=6.07\right.$, d.f. $=1, P=0.014$ ), with the red advantage seeming to decline as asymmetries in competitive ability increase (Fig. 1b). Hence, although the effect is significant for the pooled data shown in Fig. 1a, this is due principally to the results for relatively symmetric contests.

These results indicate that artificial colours may influence the outcome of physical contests in humans. A preliminary analysis of the results of the Euro 2004 international soccer tournament, in which teams wore shirts of different colours in different matches, suggests that wearing red may also bestow an advantage in team sports and when opponents wear colours other than blue. We compared the performance of five teams that wore a predominantly red shirt against their performance when wearing a different shirt colour (four played their other matches in white, one in blue). We found that all five had better results when playing in red (paired $t$-test, $t=-3.15$, d.f. $=4, P=0.034$ ), largely as a result of scoring more goals $(t=-2.98$, d.f. $=4, P=0.041)$ (further details are available from the authors). Hence, colour of sportswear may affect outcomes in a wide variety of sporting contexts.

Colour is thought to influence human mood, emotions and expressed aggression, and is a recognized element of signalling in competitive interactions in many non-human species. But it has not hitherto been suspected to be a factor in human contests. Given the ubiquity of aggressive competition throughout human societies and history, our results suggest that the evolutionary psychology of colour is likely to be a fertile field for further investigation. The implications for regulations governing sporting attire may also be important. Russell A. Hill, Robert A. Barton Evolutionary Anthropology Research Group, University of Durham, Durham DH1 3HN, UK e-mail: rahill@durhamacuk

1. Milinski, M.\& Bakkec, T. C. M. Nature 344, 330-333 (1990).

2. Wait, C.etal. Proc R. Soc Land B270, S144-S146 (2003)

3. Setchell, $\perp$ M. \& Wickings, E I.Ethalogy $111,25-50$ (2005).

4. Pryke, S, R, Andersson, S, Lawes M. I.\& Piper S.E Behav. Ecol. 13,622-631 (2002).

5. Andersson,, , Pryke, $S, R$, Omborg, 1, Lawes, M. 1 \& Andersson, M.Am. Nat. 160, 683-691 (2002).

6. Cuthill, I.C. et al. Proc. R. Soc. Lond B 264, 1093-1099 (1997).

7. Drummond,P.D. \&Quah, S.H.Psychaphysiology 38, 190-196(2001).

8. Darwin, C. The Expression of the Emotions in Man and Animals (Murray, London, 1872).

9. Drummond, P. D. Pers Indv. Diff. 23, 575-582 (1997)

10. Ryan, M. I in Behawiouval Ecology (eds Krebs, 1 R.\& Davies, N. B.) 179-202 (Blackwell Solence, Oxford, 1997).

doi: $10.1038 / 435293 a$

Supplementary information accompanies this communication on Nature's website.

Competing financial interests: dedared none. 